QUARTERLY OF APPLIED MATHEMATICS

VOLUME LXVII, NUMBER 3

SEPTEMBER 2009, PAGES 477-488

S 0033-569X(09)01148-8

Article electronically published on May 6, 2009

\title{
GLOBAL SOLUTIONS FOR COUPLED KURAMOTO-SIVASHINSKY-KDV SYSTEM
}

\author{
BY \\ MAOMAO CAI (Department of Mathematics, West Virginia University, Morgantown, WV 26506, \\ $U S A)$ \\ AND
}

DENING LI (Department of Mathematics, West Virginia University, Morgantown, WV 26506, USA)

Abstract. We study the global smooth solution for the coupled Kuramoto-Sivanshinsky-KdV system in two-dimensional space. The model is proposed to describe the surface waves on multi-layered liquid films. The global solution is obtained for general initial data, using an a priori estimate for the nonlinear system, and the smoothness of such solution is established in $t>0$.

1. Introduction. In the study of surface waves on multi-layered liquid films, the following coupled Kuramoto-Sivashinsky-Korteweg-de Vries equations are introduced (see [9] and also [6] for the two-dimensional version):

$$
\left\{\begin{array}{l}
u_{t}+u u_{x}+\Delta u_{x}=-\alpha u_{x x}-\gamma \Delta^{2} u+\epsilon_{1} v_{x} \\
v_{t}+a_{1} v_{x}=\Gamma \Delta v+\epsilon_{2} u_{x}
\end{array}\right.
$$

Here, $\Delta=\partial_{x x}+\partial_{y y}$ as usual. The coefficients $\gamma, \Gamma, \alpha, a_{1}, \epsilon_{1}, \epsilon_{2}$ in (1.1) are all positive constants.

The mixed Kuramoto-Sivashinsky-Korteweg-de Vries (KS-KdV) equation finds various applications in plasma physics, hydrodynamics and other fields; see [1, 3, 4]. The system (1.1) is a mixed KS-KdV equation, linearly coupled with an additional linear dissipative equation for an extra real wave field $v(x, y, t)$.

Received February 17, 2008.

2000 Mathematics Subject Classification. Primary 35Q53, 35Q80; Secondary 76E99.

Key words and phrases. Kuramoto-Sivashinsky-KdV system, global solution.

The first author was supported in part by DoDEPSCOR N000014-02-1-0577.

The second author was supported in part by DoDEPSCOR N000014-02-1-0577 and WVU Faculty Development Fund.

Current address: (Maomao Cai) Department of Mathematics, Weber State University, Ogden, UT 84405, USA.

E-mail address: mcai@math.wvu.edu

E-mail address: li@math.wvu.edu

(C)2009 Brown University Reverts to public domain 28 years from publication 
The one-dimensional version of (1.1) was proposed in [9] based on the KS-KdV equation for a real wave field $u(x, t)$, which is linearly coupled to an additional linear dissipative equation for an extra real wave field $v(x, t)$. The two-dimensional version is proposed in [6] in the study of cylindrical solitary pulses. One immediately notices that the two space variables $(x, y)$ in (1.1) are not symmetric. This is because of the underlying nonsymmetric physics; see [6].

Previous research by the authors of [3, 4, 5, 6, 9] on system (1.1) mainly studied the stability of the harmonic wave mode, for example, the stability of steady-state soliton solutions is analyzed by perturbation theory and wave mode in [6]. In [2], linear stability is analyzed in the context of the energy estimate and the local solution is established for the Cauchy problem to (1.1). So far, no global existence of solution for such a system is given. On the other hand, the existence of a global solution for a mixed system usually requires a stronger dissipative term, or it requires the initial data to be sufficiently small [10.

In this paper, we take advantage of the special form of the nonlinear term to derive the global estimate for a weak solution in $\eta$-weighted norms (see Theorem 3.1) and obtain smooth global solutions without the usual smallness constraints on the initial data (see Theorem 4.3). This method can also be used to establish the global existence of a class of more general systems.

Specifically, we study in this paper the global (in time) solution of (1.1) with the initial condition

$$
u(x, y, 0)=u_{0}(x, y), \quad v(x, y, 0)=v_{0}(x, y) .
$$

First, introduce some notation. Let $(\cdot, \cdot)$ denote the $L^{2}$ inner product in $R^{2}$, and let $H^{k}\left(R^{2}\right)$ be the usual Sobolev space defined by the norm

$$
\|f\|_{k}^{2}=\int_{R^{2}} \sum_{|j| \leq k}\left|D^{j} f\right|^{2} d x d y
$$

with $H^{0}\left(R^{2}\right)=L^{2}\left(R^{2}\right)$ and $\|f\|=\|f\|_{0}$ and $H^{\infty}\left(R^{2}\right)=\bigcap_{k \geq 0} H^{k}\left(R^{2}\right)$.

Let $\Pi_{T}^{k}$ be the Banach space for $(u, v):(u, v) \in \Pi_{T}^{k}$ if

$$
\begin{aligned}
& u \in C\left([0, T], H^{k+2}\left(R^{2}\right)\right) \cap L^{2}\left([0, T], H^{k+4}\left(R^{2}\right)\right), \\
& v \in C\left([0, T], H^{k+1}\left(R^{2}\right)\right) \cap L^{2}\left([0, T], H^{k+2}\left(R^{2}\right)\right) .
\end{aligned}
$$

Also, define $P_{T}$ to be the Banach space for $(u, v)$ :

$$
\begin{aligned}
& u \in C\left([0, T], H^{0}\left(R^{2}\right)\right) \cap L^{2}\left([0, T], H^{2}\left(R^{2}\right)\right), \\
& v \in C\left([0, T], H^{0}\left(R^{2}\right)\right) \cap L^{2}\left([0, T], H^{1}\left(R^{2}\right)\right) .
\end{aligned}
$$

The corresponding norm will be denoted as

$$
\|(u, v)\|_{T}^{2} \equiv \sup _{0 \leq t \leq T}\left(\|u(t)\|_{0}^{2}+\|v(t)\|_{0}^{2}\right)+\int_{0}^{T}\left(\|u(s)\|_{2}^{2}+\|v(s)\|_{1}^{2}\right) d s .
$$

The main result of this paper is the following theorem.

Theorem 1.1. Consider the initial value problem (1.1) and (1.2). 
- If initial data $\left(u_{0}, v_{0}\right) \in H^{k+2}\left(R^{2}\right) \times H^{k+1}\left(R^{2}\right)(k=0,1,2, \ldots)$, then the initial value problem (1.1), (1.2) has a unique global solution $(u, v) \in \Pi_{T}^{k}$ for all $T>0$.

- For any $k$, in particular for $k=0$, the solution $(u, v)$ is $C^{\infty}$ for $t>0$.

The paper is arranged as follows. In section 2, we derive the energy estimate for the linearized system and establish the local existence of solution for (1.1), (1.2). In section 3 , we establish an a priori $\eta$-weighted estimate for (1.1) and (1.2). In section 4 , we prove the global existence of a weak solution, and we show the global smooth solution with improved initial date. Section 4 shows that the weak solution is indeed $C^{\infty}$ in $t>0$.

2. A priori linear estimate and local existence. For the problem (1.1), (1.2), we will derive the a priori estimate for the linearized problem and then establish the local (in time) existence of the solution.

Consider the following linearized problem for (1.1), (1.2):

$$
\left\{\begin{array}{l}
u_{t}+w u_{x}+\Delta u_{x}=-\alpha u_{x x}-\gamma \Delta^{2} u+\epsilon_{1} v_{x}+f \\
v_{t}+a_{1} v_{x}=\Gamma \Delta v+\epsilon_{2} u_{x}+g \\
u(x, y, 0)=u_{0}(x, y), \quad v(x, y, 0)=v_{0}(x, y) .
\end{array}\right.
$$

We have the following:

THEOREM 2.1. Let $k \geq 0$ be an integer, and assume

- $u_{0} \in H^{k+2}\left(R^{2}\right), v_{0}(x, y) \in H^{k+1}\left(R^{2}\right)$;

- $f \in L^{2}\left([0, T], H^{k}\left(R^{2}\right)\right), g \in L^{2}\left([0, T], H^{k}\left(R^{2}\right)\right)$;

- $w \in C\left([0, T], H^{k}\left(R^{2}\right)\right) \cap L^{2}\left([0, T], H^{k+2}\left(R^{2}\right)\right)$.

Then problem (2.1) admits a unique solution $(u, v)$ in the space $\Pi_{T}^{k}$ satisfying the energy estimate

$$
\begin{aligned}
& \sup _{0 \leq t \leq T}\left(\|u(t)\|_{k+2}^{2}+\|v(t)\|_{k+1}^{2}\right) \\
& \quad+\int_{0}^{T}\left(\|u(s)\|_{k+4}^{2}+\|v(s)\|_{k+2}^{2}\right) d s \\
& \leq C_{k}\left(\left\|u_{0}\right\|_{k+2}^{2}+\left\|v_{0}\right\|_{k+1}^{2}+\int_{0}^{T}\left(\|f(s)\|_{k}^{2}+\|g(s)\|_{k}^{2}\right) d s\right) .
\end{aligned}
$$

Here $C_{k}$ is a constant depending on $T$. It depends on $w$ only in its larger norm in the spaces $C\left([0, T], H^{k}\left(R^{2}\right)\right)$ and $L^{2}\left([0, T], H^{k+2}\left(R^{2}\right)\right)$.

Proof. To establish estimate (2.2), we need only to consider smooth functions $(u, v)$.

For $k=0$, we take the $L^{2}\left(R^{2}\right)$ inner product of the equations in $(2.1)$ with $(u, v)$ and integrate by parts in the $(x, y)$-direction. A straightforward computation yields

$$
\begin{aligned}
& \partial_{t}\left(\|u(t)\|^{2}+\|v(t)\|^{2}\right)+\|u(t)\|_{2}^{2}+\|v(t)\|_{1}^{2} \\
& \quad \leq C\left(\|u(t)\|^{2}+\|v(t)\|^{2}+\|f(t)\|^{2}+\|g(t)\|^{2}\right), \quad \forall t \in[0, T] .
\end{aligned}
$$

Applying Gronwall's inequality to (2.3), we obtain further

$$
\begin{aligned}
& \sup _{0 \leq t \leq T}\left(\|u(t)\|^{2}+\|v(t)\|^{2}\right)+\int_{0}^{T}\left(\|u(s)\|_{2}^{2}+\|v(s)\|_{1}^{2}\right) d s \\
& \leq C\left(\left\|u_{0}\right\|^{2}+\left\|v_{0}\right\|^{2}+\int_{0}^{T}\left(\|f(s)\|^{2}+\|g(s)\|^{2}\right) d s\right),
\end{aligned}
$$

where $C$ always denotes a constant depending only on $T$ and coefficients of (2.1). 
Then we take the $L^{2}\left(R^{2}\right)$ inner product of the equations in (2.1) with $\left(\Delta^{2} u, \Delta v\right)$ and integrate by parts in the $(x, y)$-direction. Similarly as above and also taking into account the estimates (2.3) and (2.4), we obtain

$$
\begin{gathered}
\partial_{t}\left(\|u(t)\|_{1}^{2}+\|\Delta u\|^{2}+\|v(t)\|_{1}^{2}\right)+\|u(t)\|_{4}^{2}+\|v(t)\|_{2}^{2} \quad \forall t \in[0, T], \\
\leq C\left(\|u(t)\|_{2}^{2}+\|v(t)\|_{1}^{2}+\|f(t)\|^{2}+\|g(t)\|^{2}\right), \\
\sup _{0 \leq t \leq T}\left(\|u(t)\|_{2}^{2}+\|v(t)\|_{1}^{2}\right)+\int_{0}^{T}\left(\|u(s)\|_{4}^{2}+\|v(s)\|_{2}^{2}\right) d s \\
\leq C\left(\left\|u_{0}\right\|_{2}^{2}+\left\|v_{0}\right\|_{1}^{2}+\int_{0}^{T}\left(\|f(s)\|^{2}+\|g(s)\|^{2}\right) d s\right) .
\end{gathered}
$$

Inequality (2.6) is the $k=0$ case for (2.2). For the case $k>0$, the estimate (2.2) can be obtained by applying $\nabla_{x, y}^{k}$ to $(2.1)$ and then apply the result for $k=0$ to the expanded system.

The existence of the solution can be obtained by the continuation argument. Consider a family $(0 \leq \lambda \leq 1)$ of problems:

$$
\left\{\begin{array}{l}
u_{t}+\lambda\left[w u_{x}+\Delta u_{x}+\alpha u_{x x}+\gamma \Delta^{2} u-\epsilon_{1} v_{x}\right]+(1-\lambda) \Delta^{2} u=f \\
v_{t}+\lambda\left[a_{1} v_{x}-\Gamma \Delta v-\epsilon_{2} u_{x}\right]-(1-\lambda) \Delta v=g \\
u(x, y, 0)=u_{0}(x, y), \quad v(x, y, 0)=v_{0}(x, y)
\end{array}\right.
$$

Problem (2.1) is the case $\lambda=1$ in (2.7), while the case $\lambda=0$ in (2.7) is the well-known parabolic problem. It remains to show that the set $\mathscr{B} \subset[0,1]$ of all $\lambda$ for which (2.7) has a solution is both open and closed. This can be achieved by the fact that the solution of (2.7) satisfies (2.2) uniformly for $0 \leq \lambda \leq 1$.

- $\mathscr{B}$ is closed in $[0,1]$.

Let $\lambda_{j} \in \mathscr{B}$ and $\lambda_{j} \rightarrow \lambda_{0}$. Let $\left(u_{j}, v_{j}\right)$ be the solution of the following initial value problem:

$$
\left\{\begin{array}{l}
u_{j_{t}}+\lambda_{j} \mathscr{L}_{1}\left(u_{j}, v_{j}\right)+\left(1-\lambda_{j}\right) \Delta^{2} u_{j}=f \\
v_{j_{t}}+\lambda_{j} \mathscr{L}_{2}\left(u_{j}, v_{j}\right)-\left(1-\lambda_{j}\right) \Delta v_{j}=g \\
u_{j}(x, y, 0)=u_{0}(x, y), \quad v_{j}(x, y, 0)=v_{0}(x, y)
\end{array}\right.
$$

By $(2.2),\left(u_{j}, v_{j}\right)$ is uniformly bounded in $\Pi_{T}^{k}$. Let $\left(\bar{u}_{j}, \bar{v}_{j}\right)=\left(u_{j}-u_{j-1}, v_{j}-v_{j-1}\right)$ $(j=2,3, \cdots)$. Applying $(2.2)$ to $\left(\bar{u}_{j}, \bar{v}_{j}\right)$, we have

$$
\begin{aligned}
& \sup _{0 \leq t \leq T}\left(\left\|\bar{u}_{j}(t)\right\|_{k+2}^{2}+\left\|\bar{v}_{j}(t)\right\|_{k+1}^{2}\right) \\
& \quad+\int_{0}^{T}\left(\left\|\bar{u}_{j}(s)\right\|_{k+4}^{2}+\left\|\bar{v}_{j}(s)\right\|_{k+2}^{2}\right) d s \\
& \leq C_{k}\left|\left(\lambda_{j}-\lambda_{j-1}\right)\right|^{2} \int_{0}^{T}\left(\left\|u_{j-1}(s)\right\|_{k+4}^{2}+\left\|v_{j-1}(s)\right\|_{k+2}^{2}\right) d s \\
& \leq C_{k}\left|\left(\lambda_{j}-\lambda_{j-1}\right)\right|^{2} K .
\end{aligned}
$$

Since $\lambda_{j} \rightarrow \lambda_{0}$, it follows that $\left(u_{j}, v_{j}\right)$ is a Cauchy sequence in $\Pi_{T}^{k}$ and its limit $(u, v)$ is the solution of $(3.3)$ for $\lambda_{0}$. Hence $\mathscr{B}$ is closed in $[0,1]$.

- $\mathscr{B}$ is open in $[0,1]$.

Let $\lambda_{0} \in \mathscr{B}$ and $\lambda \in[0,1]$ with $\left|\lambda-\lambda_{0}\right| \leq \epsilon$. 
Let $\left(u_{1}, v_{1}\right)$ be the solution of the following problem:

$$
\left\{\begin{array}{l}
u_{1 t}+\lambda_{0} \mathscr{L}_{1}\left(u_{1}, v_{1}\right)+\left(1-\lambda_{0}\right) \Delta^{2} u_{1}=f \\
v_{1 t}+\lambda_{0} \mathscr{L}_{2}\left(u_{1}, v_{1}\right)-\left(1-\lambda_{0}\right) \Delta v_{1}=g \\
u_{1}(x, y, 0)=u_{0}(x, y), \quad v_{1}(x, y, 0)=v_{0}(x, y)
\end{array}\right.
$$

Let $\left(u_{j}, v_{j}\right)(j=2,3, \cdots)$ be the solution of the following problem:

$$
\left\{\begin{array}{c}
u_{j_{t}}+\lambda_{0} \mathscr{L}_{1}\left(u_{j}, v_{j}\right)+\left(1-\lambda_{0}\right) \Delta^{2} u_{j} \\
\quad=f+\left(\lambda_{0}-\lambda\right)\left(\mathscr{L}_{1}\left(u_{j-1}, v_{j-1}\right)-\Delta^{2} u_{j-1}\right), \\
v_{j_{t}}+\lambda \mathscr{L}_{2}\left(u_{j}, v_{j}\right)-\left(1-\lambda_{0}\right) \Delta v_{j} \\
=g+\left(\lambda_{0}-\lambda\right)\left(\mathscr{L}_{2}\left(u_{j-1}, v_{j-1}\right)-\Delta v_{j-1}\right), \\
u_{j}(x, y, 0)=u_{0}(x, y), \quad v_{j}(x, y, 0)=v_{0}(x, y) .
\end{array}\right.
$$

It is easy to show that for $\epsilon \ll 1,\left(u_{j}, v_{j}\right)$ is a Cauchy sequence with limit $(u, v)$ being the solution of (2.7) for $\lambda$. Hence $\mathscr{B}$ is open.

For the local solution of the nonlinear system (1.1), (1.2), we have the following:

Theorem 2.2. $\forall$ integer $k \geq 0$ and $\left(u_{0}, v_{0}\right) \in H^{k+2}\left(R^{2}\right) \times H^{k+1}\left(R^{2}\right)$ : there is a $T>0$ such that (1.1), (1.2) has a unique solution $(u, v) \in C\left([0, T] ; H^{k+2}\left(R^{2}\right) \times H^{k+1}\left(R^{2}\right)\right)$ satisfying

$$
\begin{aligned}
& \sup _{0 \leq t \leq T}\left(\|u(t)\|_{k+2}^{2}+\|v(t)\|_{k+1}^{2}\right) \\
& \quad+\int_{0}^{T}\left(\|u(s)\|_{k+4}^{2}+\|v(s)\|_{k+2}^{2}\right) d s \\
& \leq C_{k}\left(\left\|u_{0}\right\|_{k+2}^{2}+\left\|v_{0}\right\|_{k+1}^{2}\right) .
\end{aligned}
$$

In addition, the existence time span $[0, T]$ depends upon $\left(u_{0}, v_{0}\right)$ only in its norm $\left(\left\|u_{0}\right\|_{k+2}^{2}\right.$ $\left.+\left\|v_{0}\right\|_{k+1}^{2}\right)$.

Proof. The theorem is proved by linear iteration. First we construct an approximate solution $\left(u_{a}, v_{a}\right)$ by solving

$$
\left\{\begin{array}{l}
u_{t}+\Delta u_{x}+\alpha u_{x x}+\gamma \Delta^{2} u-\epsilon_{1} v_{x}=0 \\
v_{t}+a_{1} v_{x}-\Gamma \Delta v-\epsilon_{2} u_{x}=0 \\
u(x, y, 0)=u_{0}(x, y), \quad v(x, y, 0)=v_{0}(x, y) .
\end{array}\right.
$$

We look for the solution of (1.1), (1.2) in the form of $(u, v)=\left(u_{a}+\dot{u}, v_{a}+\dot{v}\right)$. The solution $(\dot{u}, \dot{v})$ is obtained by the following linear iteration $(j=1,2, \ldots)$ :

$$
\left\{\begin{array}{l}
\dot{u}_{j t}+\left(u_{a}+\dot{u}_{j-1}\right) \dot{u}_{j x}+u_{a x} \dot{u}_{j}+\Delta \dot{u}_{j x}+\alpha \dot{u}_{j x x}+\gamma \Delta^{2} \dot{u}_{j}-\epsilon_{1} \dot{v}_{j x}=-u_{a} u_{a x}, \\
\dot{v}_{j t}+a_{1} \dot{v}_{j x}-\Gamma \Delta \dot{v}_{j}-\epsilon_{2} \dot{u}_{j x}=0 \\
\dot{u}_{j}(x, y, 0)=0, \quad \dot{v}_{j}(x, y, 0)=0
\end{array}\right.
$$

By Theorem 2.1, the approximate solution $\left(u_{a}, v_{a}\right) \in \Pi_{T}^{k}$ and satisfies (2.2) with $(f, g)=0$. Since $\left(u_{a}, v_{a}\right)$ is thus fixed and $u_{a} u_{a x} \in L^{2}\left([0, T], H^{k}\left(R^{2}\right)\right)$, it follows from (2.2) that the $\left(\dot{u}_{j}, \dot{v}_{j}\right)$ is uniformly bounded in the space $\Pi_{T}^{k}$. Here $\left(\dot{u}_{j}, \dot{v}_{j}\right)$ is a Cauchy sequence from the choice of sufficiently small $T_{0}$. Such a choice of $T_{0}$ depends only upon the $\Pi_{T}^{k}$ norms of $\left(u_{a}, v_{a}\right)$ which in turn depends only on the corresponding norm of $\left(u_{0}, v_{0}\right)$. This concludes the proof of Theorem 2.2. 
To prepare for the study of the global weak solution in $P_{T}$ (see (1.4)), we need to study the linear problem under a weaker assumption on the term $f$.

Consider the following linear initial value problem:

$$
\left\{\begin{array}{l}
u_{t}+\Delta u_{x}+\alpha u_{x x}+\gamma \Delta^{2} u-\epsilon_{1} v_{x}=f_{1}, \\
v_{t}+a_{1} v_{x}-\Gamma \Delta v-\epsilon_{2} u_{x}=0, \\
u(x, y, 0)=u_{0}(x, y), v(x, y, 0)=v_{0}(x, y) .
\end{array}\right.
$$

Theorem 2.3. If $\left(u_{0}, v_{0}\right) \in L^{2}\left(R^{2}\right) \times L^{2}\left(R^{2}\right)$ and $f_{1} \in L^{1}\left([0, T] ; L^{2}\left(R^{2}\right)\right)$, then (2.15) has a unique solution $(u, v) \in P_{T}$.

Proof. Taking the $L^{2}\left(R^{2}\right)$ inner product of the two equations in (2.15) with $(u, v)$, we have

$$
\begin{aligned}
& \left(u, u_{t}\right)=\frac{1}{2} \partial_{t}\left(\|u(t)\|_{0}^{2}\right), \quad\left(v, v_{t}\right)=\frac{1}{2} \partial_{t}\left(\|v(t)\|_{0}^{2}\right) ; \\
& \left(u, \gamma \Delta^{2} u\right)=\gamma\|\Delta u\|_{0}^{2}, \quad(v,-\Gamma \Delta v)=\gamma\|\nabla v\|_{0}^{2} ; \\
& \left(u, \Delta u_{x}+\alpha u_{x x}\right) \leq\|u\|_{1}\|u\|_{2} \leq \delta\|u\|_{2}^{2}+C_{\delta}\|u\|_{1}^{2} ; \\
& \left(u, \epsilon_{1} v_{x}\right) \leq \delta\|v\|_{1}^{2}+C_{\delta}\|u\|_{0}^{2} ; \\
& \left(v, a_{1} v_{x}\right) \leq \delta\|v\|_{1}^{2}+C_{\delta}\|v\|_{0}^{2} ; \\
& \left(v, \epsilon_{2} u_{x}\right) \leq\|v\|_{0}^{2}+C\|u\|_{1}^{2} .
\end{aligned}
$$

Noticing $\|u\|_{2} \sim\|u\|_{0}+\|\Delta u\|_{0},\|v\|_{1} \sim\|v\|_{0}+\|\nabla v\|_{0}$, and $\|u\|_{1}^{2} \leq \delta\|u\|_{2}^{2}+C_{\delta}\|u\|_{0}^{2}$, we obtain by taking $\delta \ll 1$,

$$
\begin{aligned}
& \partial_{t}\left(\|u(t)\|_{0}^{2}+\|v(t)\|_{0}^{2}\right)+\|u\|_{2}^{2}+\|v\|_{1}^{2} \\
& \quad \leq C\left(\|u(t)\|_{0}^{2}+\|v(t)\|_{0}^{2}+\left\|f_{1}\right\|_{0}\|u(t)\|_{0}\right) .
\end{aligned}
$$

As in Gronwall's inequality, multiply $(2.16)$ by $e^{-C t}$ :

$$
\partial_{t}\left[e^{-C t}\left(\|u(t)\|_{0}^{2}+\|v(t)\|_{0}^{2}\right)\right]+e^{-C t}\left(\|u\|_{2}^{2}+\|v\|_{1}^{2}\right) \leq C e^{-C t}\left(\left\|f_{1}\right\|_{0}\|u(t)\|_{0}\right) .
$$

Integrating the above in $t$ yields

$$
\begin{aligned}
& \left(\|u(t)\|_{0}^{2}+\|v(t)\|_{0}^{2}\right)+\int_{0}^{t}\left(\|u\|_{2}^{2}+\|v\|_{1}^{2}\right) d s \\
& \leq C\left(\left\|u_{0}\right\|_{0}^{2}+\left\|v_{0}\right\|_{0}^{2}+\int_{0}^{t}\left\|f_{1}\right\|_{0}\|u(s)\|_{0} d s\right) .
\end{aligned}
$$

Since $\left\|f_{1}(t)\right\|_{0}$ is only in $L^{1}(0, T)$, we have

$$
\begin{aligned}
& \int_{0}^{t}\left\|f_{1}\right\|_{0}\|u(s)\|_{0} d s \leq\left(\sup _{0 \leq s \leq t}\|u(s)\|_{0}\right) \int_{0}^{t}\left\|f_{1}\right\|_{0} d s \\
& \leq \delta\left(\sup _{0 \leq s \leq t}\|u(s)\|_{0}\right)^{2}+C_{\delta}\left(\int_{0}^{t}\left\|f_{1}\right\|_{0} d s\right)^{2} .
\end{aligned}
$$

For $\delta \ll 1$, we obtain from $(2.17)$

$$
\|(u, v)\|_{T}^{2} \leq C_{T_{0}}\left(\left\|u_{0}\right\|_{0}^{2}+\left\|v_{0}\right\|_{0}^{2}+\left(\int_{0}^{T}\left\|f_{1}\right\|_{0} d s\right)^{2}\right) .
$$

Inequality (2.18) is the a priori estimate for solution $(u, v)$ of $(2.15)$. The constant $C_{T_{0}}$ in (2.18) depends on $T_{0}$ but is uniform for all $T \leq T_{0}$.

The existence of the solutions can be obtained from Theorem 2.1 and (2.18) as follows. First construct a sequence of $\left(u_{0 k}, v_{0 k}\right) \in H^{k+2}\left(R^{2}\right) \times H^{k+1}\left(R^{2}\right)$ and $f_{1 k} \in$ $L^{2}\left([0, T], H^{k}\left(R^{2}\right)\right)$ such that $\left(u_{0 k}, v_{0 k}\right) \rightarrow\left(u_{0}, v_{0}\right)$ in $H^{k+2}\left(R^{2}\right) \times H^{k+1}\left(R^{2}\right)$ and $f_{1 k} \rightarrow f_{1}$ 
in $L^{1}\left([0, T] ; L^{2}\left(R^{2}\right)\right)$. With data $\left(u_{0 k}, v_{0 k}\right)$ and $f_{1 k},(2.15)$ has a unique solution $\left(u_{k}, v_{k}\right) \in$ $\Pi_{T}^{k}$ by Theorem 2.1. Inequality (2.18) implies that $\left(u_{k}, v_{k}\right)$ is a Cauchy sequence in $\Pi_{T}^{k}$, and its limit $(u, v)$ is the required solution. This finishes the proof of Theorem 2.3.

3. Solution in $P_{T}$. First we derive a global estimate for the solutions of (1.1), (1.2) in an $\eta$-weighted norm which plays a crucial role in the proof of the global solution.

Theorem 3.1. There is an $\eta_{0}>0$ such that for any $T>0$, the solution $(u, v) \in$ $C^{1}\left([0, T], S\left(R^{2}\right)\right)$ of (1.1), (1.2) satisfies the estimate

$$
\begin{aligned}
& \sup _{0 \leq t \leq T}\left(\left\|e^{-\eta t} u(t)\right\|_{0}^{2}+\left\|e^{-\eta t} v(t)\right\|_{0}^{2}\right) \\
& \quad+\int_{0}^{T}\left(\left\|e^{-\eta s} u(s)\right\|_{2}^{2}+\left\|e^{-\eta s} v(s)\right\|_{1}^{2}\right) d s \\
& \leq C_{\eta}\left(\left\|u_{0}\right\|_{0}^{2}+\left\|v_{0}\right\|_{0}^{2}\right),
\end{aligned}
$$

$\forall \eta \geq \eta_{0}$. The constant $C_{\eta}$ in (3.1) depends only on $\eta_{0}$ and is independent of $T$.

Proof. Let $(\tilde{u}, \tilde{v})=\left(e^{-\eta t} u, e^{-\eta t} v\right)$; then $(\tilde{u}, \tilde{v})$ satisfies the following:

$$
\left\{\begin{array}{l}
\tilde{u}_{t}+e^{\eta t} \tilde{u} \tilde{u}_{x}+\Delta \tilde{u}_{x}+\eta \tilde{u}=-\alpha \tilde{u}_{x x}-\gamma \Delta^{2} \tilde{u}+\epsilon_{1} \tilde{v}_{x}, \\
\tilde{v}_{t}+a_{1} \tilde{v}_{x}+\eta \tilde{v}=\Gamma \Delta \tilde{v}+\epsilon_{2} \tilde{u}_{x}, \\
\tilde{u}(x, y, 0)=u_{0}(x, y), \tilde{v}(x, y, 0)=v_{0}(x, y) .
\end{array}\right.
$$

Take the inner product of the two equations in $(3.2)$ with $(\tilde{u}, \tilde{v})$. Since the only nonlinear term

$$
\left(e^{\eta t} \tilde{u} \tilde{u}_{x}, \tilde{u}\right)=0
$$

and since we have on the left-hand side of the estimate the terms $\eta\|\tilde{u}\|^{2}+\eta\|\tilde{v}\|^{2}$, we can then estimate all the remaining terms as in the case of the linear problem by choosing $\eta_{0} \gg 1$.

Remark 3.1. For functions $(u, v) \in P_{T}$, since the dual of $u u_{x}$ with $u$ is well-defined, we conclude that the estimate (3.1) is also valid for solution $(u, v) \in P_{T}$.

The following theorem establishes the uniqueness of solution $(u, v) \in P_{T}$ for (1.1), (1.2).

Theorem 3.2. The solution $(u, v) \in P_{T}$ of (1.1), (1.2) is unique.

Proof. Let $\left(u_{1}, v_{1}\right)$ and $\left(u_{2}, v_{2}\right)$ be two solutions in $P_{T}$ for (1.1), (1.2). Since

$$
u_{1} u_{1 x}-u_{2} u_{2 x}=u_{1 x} \hat{u}+u_{2} \hat{u}_{x}
$$

then $(\hat{u}, \hat{v}) \equiv\left(u_{1}-u_{2}, v_{1}-v_{2}\right)$ satisfies the following linear problem:

$$
\left\{\begin{array}{l}
\hat{u}_{t}+u_{1 x} \hat{u}+u_{2} \hat{u}_{x}+\Delta \hat{u}_{x}=-\alpha \hat{u}_{x x}-\gamma \Delta^{2} \hat{u}+\epsilon_{1} \hat{v}_{x}, \\
\hat{v}_{t}+a_{1} \hat{v}_{x}=\Gamma \Delta \hat{v}+\epsilon_{2} \hat{u}_{x} \\
\hat{u}(x, y, 0)=0, \quad \hat{v}(x, y, 0)=0 .
\end{array}\right.
$$

Because $H^{2}\left(R^{2}\right)$ is a Banach algebra,

$$
\left(u_{1 x} \hat{u}, \hat{u}\right) \leq\left\|u_{1 x}\right\|\left\|\hat{u}^{2}\right\|_{0} \leq C\left\|u_{1}\right\|_{1}\|\hat{u}\|_{0}^{2} .
$$


Also by the Sobolev imbedding theorem, we have

$$
\left(u_{2} \hat{u}_{x}, \hat{u}\right) \leq \sup _{x, y}\left|u_{2}\right|\|\hat{u}\|_{1}^{2} \leq C\left\|u_{2}\right\|_{2}\|\hat{u}\|_{1}^{2} \leq \epsilon\|\hat{u}\|_{2}^{2}+C(\epsilon)\|\hat{u}\|_{0}^{2} .
$$

Since $u_{1}, u_{2}$ are fixed functions in $P_{T}$, we can choose $\epsilon \ll 1$ such that the solution for linear problem (3.5) satisfies the estimate (2.18) in Theorem 2.3. Since $f_{1}=0$ and $(\hat{u}(0), \hat{v}(0))=0$, we have $(\hat{u}, \hat{v})=0$. This concludes the proof of Theorem 3.2.

The following local existence theorem is similar to Theorem 2.2, except that the regularity condition on the initial data $\left(u_{0}, v_{0}\right)$ is weaker and the solution $(u, v)$ is also weaker in regularity.

Theorem 3.3. Let $\left(u_{0}, v_{0}\right) \in L^{2}\left(R^{2}\right) \times L^{2}\left(R^{2}\right)$. Then there is a $T_{0}>0$ such that (1.1), (1.2) has a unique solution $(u, v) \in P_{T_{0}}$ satisfying

$$
\begin{aligned}
& \sup _{0 \leq t \leq T}\left(\|u(t)\|_{0}^{2}+\|v(t)\|_{0}^{2}\right)+\int_{0}^{T}\left(\|u(s)\|_{2}^{2}+\|v(s)\|_{1}^{2}\right) d s \\
& \leq C_{T}\left(\left\|u_{0}\right\|_{0}^{2}+\left\|v_{0}\right\|_{0}^{2}\right) .
\end{aligned}
$$

In particular, the existence time span $\left[0, T_{0}\right]$ depends upon $\left(u_{0}, v_{0}\right)$ only in its norm $\left(\left\|u_{0}\right\|_{0}^{2}+\left\|v_{0}\right\|_{0}^{2}\right)$.

Proof. As in the proof of Theorem 2.2, we first construct an approximate solution $\left(u_{a}, v_{a}\right)$ by solving $(2.5)$. Also, $\left(u_{a}, v_{a}\right)$ satisfies the estimate

$$
\begin{gathered}
\sup _{0 \leq t \leq T}\left(\left\|u_{a}(t)\right\|_{0}^{2}+\left\|v_{a}(t)\right\|_{0}^{2}\right)+\int_{0}^{T}\left(\left\|u_{a}(s)\right\|_{2}^{2}+\left\|v_{a}(s)\right\|_{1}^{2}\right) d s \\
\leq C_{T}\left(\left\|u_{0}\right\|_{0}^{2}+\left\|v_{0}\right\|_{0}^{2}\right) .
\end{gathered}
$$

In particular, we notice that $u_{a} u_{a x} \in L^{1}\left((0, T) ; L^{2}\left(R^{2}\right)\right)$ with

$$
\int_{0}^{T}\left\|u_{a} u_{a x}\right\|_{0} d s \leq \int_{0}^{T}\left\|u_{a}(s)\right\|_{2}^{2} d s \leq C_{T}\left(\left\|u_{0}\right\|_{0}^{2}+\left\|v_{0}\right\|_{0}^{2}\right)^{2} .
$$

We again look for the solution of (1.1), (1.2) in the form of $(u, v)=(\dot{u}, \dot{v})$. Obviously, $(u, v)$ is a solution of (1.1), (1.2) if and only if $\left(u_{a}+\dot{u}, v_{a}+\dot{v}\right)$ satisfies

$$
\left\{\begin{array}{l}
\dot{u}_{t}+\left(u_{a}+\dot{u}\right) \dot{u}_{x}+u_{a x} \dot{u}+\Delta \dot{u}_{x}+\alpha \dot{u}_{x x}+\gamma \Delta^{2} \dot{u}-\epsilon_{1} \dot{v}_{x}=-u_{a} u_{a x}, \\
\dot{v}_{t}+a_{1} \dot{v}_{x}-\Gamma \Delta \dot{v}-\epsilon_{2} \dot{u}_{x}=0, \\
\dot{u}(x, y, 0)=0, \quad \dot{v}(x, y, 0)=0 .
\end{array}\right.
$$

The solution $(\dot{u}, \dot{v})$ of $(3.11)$ is obtained by the linear iteration $(j=1,2, \ldots)$ :

$$
\left\{\begin{array}{l}
\dot{u}_{j t}+\left(u_{a}+\dot{u}_{j-1}\right) \dot{u}_{j x}+u_{a x} \dot{u}_{j}+\Delta \dot{u}_{j x}+\alpha \dot{u}_{j x x}+\gamma \Delta^{2} \dot{u}_{j}-\epsilon_{1} \dot{v}_{j x} \\
\quad=-u_{a} u_{a x} \\
\dot{v}_{j t}+a_{1} \dot{v}_{j x}-\Gamma \Delta \dot{v}_{j}-\epsilon_{2} \dot{u}_{j x}=0 \\
\dot{u}_{j}(x, y, 0)=0, \quad \dot{v}_{j}(x, y, 0)=0
\end{array}\right.
$$

with $\left(\dot{u}_{0}, \dot{v}_{0}\right)=(0,0)$.

We are going to show that the following.

(1) For any $\kappa>0$, we can choose $T_{0} \ll 1$ such that for all $j$ :

$$
\left\|\left(\dot{u}_{j}, \dot{v}_{j}\right)\right\|_{T_{0}}^{2} \leq \kappa .
$$


(2) There is a $T_{1} \leq T_{0}$ such that for all $j \geq 1$ :

$$
\left\|\left(\dot{u}_{j}, \dot{v}_{j}\right)\right\|_{T_{1}} \leq \frac{1}{2} \mid\left\|\left(\dot{u}_{j-1}, \dot{v}_{j-1}\right)\right\|_{T_{1}} .
$$

Obviously, (3.13) and (3.14) imply the existence of a local solution $(u, v) \in P_{T_{1}}$, as claimed in Theorem 3.3.

- Prove (3.13):

Assume $\left\|\left(\dot{u}_{j-1}, \dot{v}_{j-1}\right)\right\|_{T} \leq \kappa$ and consider the energy estimate for $\left(\dot{u}_{j}, \dot{v}_{j}\right)$. We have

$$
\left\{\begin{array}{l}
\left(u_{a} \dot{u}_{j x}, \dot{u}_{j}\right) \leq\left\|\dot{u}_{j}\right\|_{0}\left\|u_{a}\right\|_{2}\left\|\dot{u}_{j}\right\|_{2} \leq C_{\delta}\left\|\dot{u}_{j}(t)\right\|_{0}^{2}+\delta\left\|\dot{u}_{j}\right\|_{2}^{2}, \\
\left(u_{a x} \dot{u}_{j}, \dot{u}_{j}\right) \leq\left\|\dot{u}_{j}\right\|_{0}\left\|u_{a}\right\|_{2}\left\|\dot{u}_{j}\right\|_{2} \leq C_{\delta}\left\|\dot{u}_{j}(t)\right\|_{0}^{2}+\delta\left\|\dot{u}_{j}\right\|_{2}^{2}, \\
\left(\dot{u}_{j-1} \dot{u}_{j x}, \dot{u}_{j}\right) \leq\left\|\dot{u}_{j}\right\|_{0}\left\|\dot{u}_{j-1}\right\|_{2}\left\|\dot{u}_{j}\right\|_{2} \leq C_{\delta}\left\|\dot{u}_{j}(t)\right\|_{0}^{2}+\delta\left\|\dot{u}_{j}\right\|_{2}^{2} .
\end{array}\right.
$$

In particular, the constant $C_{\delta}$ in (3.15) depends only on $\kappa$ and $\left|\left\|\left(u_{a}, v_{a}\right)\right\|\right|_{T}$ and is independent of specific $\dot{u}_{j-1}$.

Choosing $\delta$ in (3.15) sufficiently small, we can obtain an energy estimate for $\left(\dot{u}_{j}, \dot{v}_{j}\right)$ similar to $(2.10)$ :

$$
\left\|\left(\dot{u}_{j}, \dot{v}_{j}\right)\right\|_{T}^{2} \leq C_{T}\left(\int_{0}^{T}\left\|u_{a} u_{a x}\right\|_{0} d s\right)^{2} .
$$

Since the constant $C_{T}$ in (3.16) is uniform for $T<1$, we can choose $T_{0} \ll 1$ so that

$$
C_{T}\left(\int_{0}^{T_{0}}\left\|u_{a} u_{a x}\right\|_{0} d s\right)^{2} \leq \kappa .
$$

Here, $T_{0}$ depends only on the $L^{1}(0, T)$ norm of $\left\|u_{a} u_{a x}\right\|_{0}$ which in turn depends on $\left\|u_{0}\right\|_{0}+\left\|v_{0}\right\|_{0}$ by (3.10). This concludes the proof of (3.13).

- Prove (3.14):

For $T_{0}$ chosen above and $\forall T \leq T_{0}$, let $\left(\tilde{u}_{j}, \tilde{v}_{j}\right)=\left(\dot{u}_{j}-\dot{u}_{j-1}, \dot{v}_{j}-\dot{v}_{j-1}\right)$. Then from $(3.12),\left(\tilde{u}_{j}, \tilde{v}_{j}\right)$ satisfies

$$
\left\{\begin{array}{l}
\tilde{u}_{j t}+\Delta \tilde{u}_{j x}+\alpha \tilde{u}_{j x x}+\gamma \Delta^{2} \tilde{u}_{j}-\epsilon_{1} \tilde{v}_{j x} \\
\quad+u_{a} \tilde{u}_{j x}+u_{a x} \tilde{u}_{j}=\dot{u}_{j-2} \dot{u}_{(j-1) x}-\dot{u}_{j-1} \dot{u}_{j x} \\
\tilde{v}_{j t}+a_{1} \tilde{v}_{j x}-\Gamma \Delta \tilde{v}_{j}-\epsilon_{2} \tilde{u}_{j x}=0 \\
\tilde{u}_{j}(x, y, 0)=0, \tilde{v}_{j}(x, y, 0)=0 .
\end{array}\right.
$$

Because

$$
\dot{u}_{j-2} \dot{u}_{(j-1) x}-\dot{u}_{j-1} \dot{u}_{j x}=-\dot{u}_{j-1} \tilde{u}_{j x}-\dot{u}_{(j-1) x} \tilde{u}_{j-1},
$$

similarly to (3.15), we have

$$
\left\{\begin{array}{l}
\left(\dot{u}_{j-1} \tilde{u}_{j x}, \tilde{u}_{j}\right) \leq \kappa\left\|\tilde{u}_{j}\right\|_{2}^{2} \\
\left(\dot{u}_{(j-1) x} \tilde{u}_{j-1}, \tilde{u}_{j}\right) \leq \kappa\left(\left\|\tilde{u}_{j-1}\right\|_{0}^{2}+\left\|\tilde{u}_{j}\right\|_{2}^{2}\right) .
\end{array}\right.
$$


Then as in (3.16), we obtain

$$
\begin{aligned}
& \left\|\left(\tilde{u}_{j}, \tilde{v}_{j}\right)\right\|_{T}^{2} \leq C_{T}\left(\int_{0}^{T}\left\|\dot{u}_{j-1} \tilde{u}_{j x}\right\|_{0} d s+\int_{0}^{T}\left\|\dot{u}_{(j-1) x} \tilde{u}_{j-1}\right\|_{0} d s\right)^{2} \\
& \leq C_{T}\left(\kappa \int_{0}^{T}\left\|\tilde{u}_{j}\right\|_{2}^{2} d s+\kappa \int_{0}^{T}\left\|\tilde{u}_{j-1}\right\|_{2}^{2} d s\right) .
\end{aligned}
$$

Notice $C_{T}$ is uniform for $T \leq T_{0}$. We can choose $T_{0} \ll 1$ (by (3.13)) so that $\kappa \ll 1$ such that $C_{T} \kappa \leq \frac{1}{4}$. For such chosen $T_{1}$, (3.14) follows readily from (3.20). This concludes the proof of Theorem 3.3.

Theorem 3.4 (Global existence of the $P_{T}$ solution). Let $\left(u_{0}, v_{0}\right) \in L^{2}\left(R^{2}\right) \times L^{2}\left(R^{2}\right)$. Then $\forall T>0,(1.1),(1.2)$ has a unique solution $(u, v) \in P_{T}$ satisfying

$$
\begin{aligned}
& \sup _{0 \leq t \leq T}\left(\|u(t)\|_{0}^{2}+\|v(t)\|_{0}^{2}\right)+\int_{0}^{T}\left(\|u(s)\|_{2}^{2}+\|v(s)\|_{1}^{2}\right) d s \\
& \leq C_{T}\left(\left\|u_{0}\right\|_{0}^{2}+\left\|v_{0}\right\|_{0}^{2}\right) .
\end{aligned}
$$

Proof. Let $\Lambda$ be the subset of all $T \geq 0$ such that in $[0, T]$, the problem (1.1), (1.2) has a unique solution in $P_{\tau}$. Theorem 3.3 guarantees that $\Lambda \neq \emptyset$. Since $\forall T \in \Lambda$, $(u(T), v(T)) \in L^{2}\left(R^{2}\right)$, Theorem 3.3 also implies that the set $\Lambda$ must be open. Let $\left\{T_{k}\right\}$ be a monotone increasing sequence in $\Lambda$ such that $T_{k} \rightarrow T_{\tau}$. By Theorem 3.1, $\left(u\left(T_{\tau}\right), v\left(T_{\tau}\right)\right) \in L^{2}\left(R^{2}\right)$. Then there must be a solution in $\left[0, T_{\tau}+\epsilon\right]$ by Theorem 3.3. Hence $\Lambda$ must be open. Consequently $\Lambda=[0, \infty)$ and this concludes the proof of Theorem 3.4 .

4. Global smooth solutions. Let $(u, v) \in P_{T}$ be the global solution obtained in Theorem 3.4. Define $f_{1}=-u u_{x}$. Then $f_{1} \in L^{1}\left([0, T] ; H^{1}\left(R^{2}\right)\right)$ and

$$
\int_{0}^{T}\left\|f_{1}\right\|_{1} d t \leq \int_{0}^{T}\|u\|_{2}^{2} d t \leq C_{T}\left(\left\|u_{0}\right\|_{0}^{2}+\left\|v_{0}\right\|_{0}^{2}\right) .
$$

Consider the following linear initial value problem:

$$
\left\{\begin{array}{l}
u_{t}+\Delta u_{x}+\alpha u_{x x}+\gamma \Delta^{2} u-\epsilon_{1} v_{x}=f_{1}, \\
v_{t}+a_{1} v_{x}-\Gamma \Delta v-\epsilon_{2} u_{x}=0, \\
u(x, y, 0)=u_{0}(x, y), \quad v(x, y, 0)=v_{0}(x, y) .
\end{array}\right.
$$

Then we have the following improved version of Theorem 2.3 .

Theorem 4.1. If $\left(u_{0}, v_{0}\right) \in H^{2}\left(R^{2}\right) \times H^{1}\left(R^{2}\right)$ and $f_{1} \in L^{1}\left([0, T] ; H^{1}\left(R^{2}\right)\right)$, then (4.2) has a unique solution $(u, v) \in \Pi_{T}^{0}$.

Proof. Taking the $L^{2}$ inner product over $R^{2}$ of the equations in (4.2) with $(\Delta u, \Delta v)$ and noticing $\left(\Delta u_{x}, \Delta u\right)=0$, we obtain readily the following:

$$
\begin{gathered}
\partial_{t}\left(\|\nabla u\|^{2}+\|\nabla v\|^{2}\right)+\gamma\|\nabla \Delta u\|^{2}+\Gamma\|\nabla v\|^{2} \\
\leq C\left(\|u\|_{2}^{2}+\|v\|_{1}^{2}+\left|\left(\nabla f_{1}, \nabla u\right)\right|\right) .
\end{gathered}
$$


Integrating (4.3) from 0 to $T$ and noticing $(u, v) \in P_{T}$ and satisfies (3.21), we have

$$
\begin{aligned}
& \sup _{0 \leq t \leq T}\left(\|u(t)\|_{1}^{2}+\|v(t)\|_{1}^{2}\right)+\int_{0}^{T}\left(\|u(s)\|_{3}^{2}+\|v(s)\|_{2}^{2}\right) d s \\
& \quad \leq C\left(\left\|u_{0}\right\|_{1}^{2}+\left\|v_{0}\right\|_{1}^{2}+\int_{0}^{T}\left|\left(\nabla f_{1}, \nabla u\right)\right| d s\right)
\end{aligned}
$$

since

$$
\begin{aligned}
& \int_{0}^{T}\left|\left(\nabla f_{1}, \nabla u\right)\right| d s \leq \int_{0}^{T}\left\|\nabla f_{1}\right\|_{0}\|\nabla u(s)\|_{0} d s \\
& \quad \leq \sup _{0 \leq t \leq T}\|u(s)\|_{1} \int_{0}^{T}\left\|f_{1}\right\|_{1} d s \\
& \quad \leq \delta \sup _{0 \leq t \leq T}\|u(s)\|_{1}^{2}+C_{\delta}\left(\int_{0}^{T}\left\|f_{1}\right\|_{1} d s\right)^{2} .
\end{aligned}
$$

By (4.1), $\left(\int_{0}^{T}\left\|f_{1}\right\|_{1} d s\right)$ is bounded by $\left\|u_{0}\right\|_{0}^{2}+\left\|v_{0}\right\|_{0}^{2}$. Combining (4.4) and (4.5), we obtain

$$
\begin{aligned}
& \sup _{0 \leq t \leq T}\left(\|u(t)\|_{1}^{2}+\|v(t)\|_{1}^{2}\right)+\int_{0}^{T}\left(\|u(s)\|_{3}^{2}+\|v(s)\|_{2}^{2}\right) d s \\
& \quad \leq C_{T}\left(\left\|u_{0}\right\|_{1}^{2}+\left\|v_{0}\right\|_{1}^{2}+\left(\int_{0}^{T}\left\|f_{1}\right\|_{1} d s\right)^{2}\right) .
\end{aligned}
$$

Inequality (4.6) implies that for $\left(u_{0}, v_{0}\right) \in H^{1}\left(R^{2}\right)$, the solution $(u, v)$ in Theorem 3.4 is actually in the space

$$
\begin{aligned}
& u \in C\left([0, T] ; H^{1}\right) \bigcap L^{2}\left([0, T] ; H^{3}\right), \\
& v \in C\left([0, T] ; H^{1}\right) \bigcap L^{2}\left([0, T] ; H^{2}\right) .
\end{aligned}
$$

A further consequence of (4.7) is that $f_{1}=u u_{x} \in L^{2}\left((0, T) \times R^{2}\right)$.

Taking the $L^{2}$ inner product over $R^{2}$ of the first equation in (4.2) with $\Delta^{2} u$, we further obtain

$$
\partial_{t}\|\Delta u\|^{2}+\gamma\left\|\Delta^{2} u\right\|^{2} \leq C\left(\|u\|_{3}^{2}+\|v\|_{1}^{2}+\int_{0}^{T}\left\|f_{1}\right\|_{0}^{2} d t\right) .
$$

Combining (4.6) and (4.8), we obtain that the solution $(u, v)$ in Theorem 3.4 is in $\Pi_{T}^{0}$. This completes the proof of Theorem 4.1.

For any integer $k>0$, we have the following:

Theorem 4.2. Assume that in (1.1), (1.2), $\left(u_{0}, v_{0}\right) \in H^{k+2}\left(R^{2}\right) \times H^{k+1}\left(R^{2}\right)$. Then the global solution $(u, v)$ in Theorem 3.4 is actually $\in \Pi_{T}^{k}$.

Proof. Theorem 4.2 can be proved by induction. If the solution $(u, v) \in \Pi_{T}^{k-1}$, then $u \in C\left((0, T) ; H^{k+1}\right) \cap L^{2}\left((0, T) ; H^{k+3}\right)$ and $\nabla^{k}\left(u u_{x}\right) \in L^{2}\left((0, T) \times R^{2}\right)$. Therefore we can apply $\nabla^{k}$ to (1.1), (1.2) and the result obtained in Theorem 4.1 to $\left(\nabla^{k} u, \nabla^{k} v\right)$. Theorem 4.2 follows readily.

REMARK 4.1. Since integer $k$ in Theorem 4.2 is arbitrary, Theorem 4.2 also implies that for smooth initial data $\left(u_{0}, v_{0}\right)$, the unique global solution $(u, v)$ is also smooth.

The global smooth solution in Theorem 4.2 is obtained under the assumption that the initial data $\left(u_{0}, v_{0}\right)$ is sufficiently smooth. Actually we can drop such conditions on $\left(u_{0}, v_{0}\right)$ and still obtain the smoothness of the solution $(u, v)$ for $t>0$.

Theorem 4.3. In the problem (1.1), (1.2), assume $\left(u_{0}, v_{0}\right) \in L^{2}\left(R^{2}\right) \times L^{2}\left(R^{2}\right)$. Then the unique solution $(u, v) \in P_{T}$ obtained in Theorem 3.4 is actually in $C^{\infty}\left((0, T) \times R^{2}\right)$. 
Proof. Let $\phi_{\epsilon}(t) \in C^{\infty}[0, \infty)$ such that

$$
\phi_{\epsilon}(t)=\left\{\begin{array}{l}
0, \quad 0 \leq t \leq \epsilon ; \\
\text { monnotone increasing, } \epsilon \leq t \leq 2 \epsilon ; \\
1,2 \epsilon \leq t
\end{array}\right.
$$

Let

$$
\left(u_{\epsilon}, v_{\epsilon}\right)=\left(u \phi_{\epsilon}(t), v \phi_{\epsilon}(t)\right) .
$$

Since $(u, v)$ and $\left(u_{\epsilon}, v_{\epsilon}\right)$ are identical for $t \geq 2 \epsilon$ and $\epsilon$ is arbitrary, we need only to show that $\left(u_{\epsilon}, v_{\epsilon}\right) \in C^{\infty}\left((0, T) \times R^{2}\right)$.

$\left(u_{\epsilon}, v_{\epsilon}\right)$ satisfies the following linear initial value problem:

$$
\left\{\begin{array}{l}
u_{\epsilon t}+\Delta u_{\epsilon x}+\alpha u_{\epsilon x x}+\gamma \Delta^{2} u_{\epsilon}-\epsilon_{1} v_{\epsilon x}=f, \\
v_{\epsilon t}+a_{1} v_{\epsilon x}-\Gamma \Delta v_{\epsilon}-\epsilon_{2} u_{\epsilon x}=g \\
u_{\epsilon}(x, y, 0)=0, \quad v_{\epsilon}(x, y, 0)=0
\end{array}\right.
$$

In (4.11),

$$
\begin{aligned}
& f=-\phi_{\epsilon t} u-\phi_{\epsilon} u u_{x} \in L^{1}\left((0, T), H^{1}\left(R^{2}\right)\right), \\
& g=-\phi_{\epsilon t} v \in L^{2}\left((0, T), H^{1}\left(R^{2}\right)\right) .
\end{aligned}
$$

Similarly to the proof of Theorem 2.1 and Theorem 4.1, we can derive the estimate for the solution of (4.11), $\left(u_{\epsilon}, v_{\epsilon}\right) \in \Pi_{T}^{0}$. This would imply that $(f, g) \in L^{2}\left((0, T), H^{1}\left(R^{2}\right)\right)$. Applying the result of Theorem 2.1 again, we have $\left(u_{\epsilon}, v_{\epsilon}\right) \in \Pi_{T}^{1}$ and hence $(f, g) \in$ $L^{2}\left((0, T), H^{2}\left(R^{2}\right)\right)$ and so on. Consequently we obtain that $\forall k,\left(u_{\epsilon}, v_{\epsilon}\right) \in \Pi_{T}^{k}$. This implies that $\left(u_{\epsilon}, v_{\epsilon}\right) \in L^{2}\left((0, T) ; C^{\infty}\left(R^{2}\right)\right)$. From the fact that $\left(u_{\epsilon}, v_{\epsilon}\right)$ satisfies the differential equation (4.11), we obtain the smoothness of $\left(u_{\epsilon}, v_{\epsilon}\right)$ in the $t$-direction; hence $\left(u_{\epsilon}, v_{\epsilon}\right) \in C^{\infty}\left((0, T) \times R^{2}\right)$. This concludes the proof of Theorem 4.3.

\section{REFERENCES}

[1] D. J. Benney, Long waves on liquid films, J. Math. Phys. 45(1966), 150-155. MR0201125 (34:1010)

[2] M. Cai, D. Li and C. Rattanakul, Stability and existence of solutions for 2-dimensional coupled Kuramoto-Sivashinsky-KdV system (preprint).

[3] C. I. Christov and M. G. Velarde, Dissipative solitons, Physica D, 86(1995), 323-347. MR1353963 (96h:35178)

[4] C. Elphick, G. R. Ierlev, O. Regev and E. A. Spiegel, Interacting localized structures with Galilean invariance, Phys. Rev. A, 44(1991), 1110-1122.

[5] B. F. Feng, B. A. Malomed and T. Kawahara, Stable periodic waves in coupled KuramotoSivashinsky-Korteweg-de Vries equations, J. Phys. Soc. Jpn. 71 (2002), 2700-2707.

[6] B. F. Feng, B. A. Malomed and T. Kawahara, Cylindrical solitary pulses in a two-dimensional stabilized Kuramoto-Sivashinsky system, Physica D, 3035 (2002), 1-12. MR.1963855 (2004b:35282)

[7] J. A. Gear and R. Grimshaw, Weak and strong interactions between internal solitary waves, Stud. Appl. Math. 70(1984), 235-258. MR742590(85i:76013)

[8] T. Kato, The Cauchy problem for quasilinear symmetric hyperbolic systems, Arch. Rat. Mech. Anal. 58(1975), 181-205. MR0390516 (52:11341)

[9] B. A. Malomed, B. F. Feng and T. Kawahara, Stabilized Kuramoto-Sivashinsky system, Phys. Rev. E 64, 046304(2001)

[10] A. Matsumura and T. Nishida, The initial value problem for the equations of motion of viscous and heat-conductive gases, J. Math. Kyoto Univ. 20-1 (1980), 67-104. MR.564670 (81g:35108)

[11] M. Renardy and R. Rogers, An introduction to partial differential equations, Springer-Verlag, New York, 2004. MR2028503 (2004j:35001) 